\title{
Mapping of moveout approximations in TI media
}

A. Stovas*, NTNU Norway and T. Alkhalifah, KAUST Saudi Arabia

\section{Summary}

Moveout approximations play very important role in seismic modeling, inversion and scanning for parameters in complex media. We propose to map the moveout approximations from VTI to TTI medium by introducing the effective tilt angle. As a result, we obtain highly accurate TTI moveout equations synonymous with their VTI counterparts.

\section{Introduction}

The theory of moveout approximations in anisotropic media with a tilted symmetry axis (TTI medium) is developed in recent years (Pech, Tsvankin and Grechka, 2003; Alkhalifah, 2011b). In order to derive the moveout approximation in a TTI medium, Stovas and Alkhalifah (2011) used the perturbation method with anelliptic parameter $\eta$ being a small parameter. They derive a trial solution of the TTI eikonal equation, and applied the Shanks transform in order to stabilize the truncated power series. Generally, the presence of tilt results in significant complications in moveout expressions and traveltime parameters.

In this abstract, we use the moveout mapping concept proposed earlier by the authors (Stovas and Alkhalifah, 2012) to derive the TTI moveout approximations from the ones defined for a VTI medium.

\section{The moveout mapping in TI media}

In a homogeneous 2D TI medium, the mapping of moveout from VTI to TTI model is given by rational equations (Stovas and Alkhalifah, 2012)

$$
\begin{aligned}
& x_{n}(p)=z \frac{x(p) \cos \theta+z \sin \theta}{z \cos \theta-x(p) \sin \theta} \\
& t_{n}(p)=t(p) \frac{z}{z \cos \theta-x(p) \sin \theta}
\end{aligned}
$$

where $(x, t)$ and $\left(x_{n}, t_{n}\right)$ are the offset-traveltime points in a VTI and TTI model, respectively, $p$ is the horizontal slowness in a VTI model, $\mathrm{z}$ is the layer thickness and $\theta$ is the tilt angle. One can see that $\left(x=0, t=t_{0}\right) \Leftrightarrow\left(x_{n}=z \tan \theta, t_{n}=t_{0} / \cos \theta\right) \quad$ and $\left(x=-z \tan \theta, t=t_{n 0} / \cos \theta\right) \Leftrightarrow\left(x_{n}=0, t_{n}=t_{n 0}\right)$. The inverse transform is defined as

$$
\begin{aligned}
& x(p)=z \frac{x_{n}(p) \cos \theta-z \sin \theta}{z \cos \theta+x_{n}(p) \sin \theta} \\
& t(p)=t_{n}(p) \frac{z}{z \cos \theta+x_{n}(p) \sin \theta}
\end{aligned}
$$

The function $t_{n}\left(x_{n}\right)$ is no longer symmetric and has a minimum at $x_{n}=x_{n 0}$, which is defined by equation $d t_{n} / d x_{n}=0$ with two solutions: $d x / d p=0$ (the caustic condition) and $p_{n}=p \cos \theta+q \sin \theta=0 \quad$ (the zero horizontal slowness in $p_{n}, q_{n}$ space).

\section{Mapping of moveout approximations}

There exists many different moveout approximations developed recently (Malovichko, 1978; Alkhalifah and Tsvankin, 1995; Fomel and Stovas, 2010; Alkhalifah, 2011a). To derive the moveout approximation for TTI media is non-trivial task. Recently, Alkhalifah (2011a) and Stovas and Alkhalifah (2011) proposed the moveout approximations based on the linearization of eikonal equation in $\theta$ and in $\eta$, respectively, and following use of the Shanks transform, which allowed for an inhomogeneous background medium treatment.

Another approach, we propose in this abstract, is to map the moveout approximation from a VTI to TTI medium based on the mapping equations (1). We start with an approximation for a VTI medium in the form $t(x)$ as a function of also the vertical traveltime $t_{0}$, the normal moveout velocity $v_{n}$ and the anelliptic parameter $\eta$. Using equation (1), we obtain a generalized approach to transform VTI approximations to TTI ones using the following formula,

$t_{n}\left(x_{n}\right)=t\left(x=z \frac{x_{n} \cos \theta-z \sin \theta}{z \cos \theta+x_{n} \sin \theta}\right)\left(\cos \theta+\frac{x_{n}}{z} \sin \theta\right)$.

Let us illustrate the transformation (3) on a few known moveout approximations. In all following equations, we will use $z=v_{0} t_{0}$.

The Taylor series for traveltime squared that is given by

$t^{2}(x)=t_{0}^{2}+\frac{x^{2}}{v_{n}^{2}}-\frac{2 \eta x^{4}}{v_{n}^{4} t_{0}^{2}}+\ldots$ 


\section{Mapping of moveout approximations in TI media}

transforms, using equation 3 , into the series

$$
\begin{aligned}
& t_{n}^{2}\left(x_{n}\right)=t_{0}^{2} \cos ^{2} \theta\left[1+\frac{v_{0}^{2}}{v_{n}^{2}} \tan ^{2} \theta-\frac{2 \eta v_{0}^{4}}{v_{n}^{4}} \tan ^{4} \theta\right] \\
& +\frac{v_{0} t_{0} x_{n}}{v_{n}^{2}}\left[\left(\frac{v_{n}^{2}}{v_{0}^{2}}-1\right) \sin 2 \theta+2 \eta \frac{v_{0}^{2}}{v_{n}^{2}}(3+\cos 2 \theta) \tan ^{3} \theta\right] \\
& +\frac{x_{n}^{2}}{v_{n}^{2}}\left[\cos ^{2} \theta+\sin ^{2} \theta\left(\frac{v_{n}^{2}}{v_{0}^{2}}-2 \eta \frac{v_{0}^{2}}{v_{n}^{2}}\left(6+8 \tan ^{2} \theta+3 \tan ^{4} \theta\right)\right)\right] \\
& +\frac{8 \eta v_{0} \tan \theta}{t_{0} v_{n}^{4} \cos ^{4} \theta} x_{n}^{3} \\
& -\frac{2 \eta(3-2 \cos 2 \theta) x_{n}^{4}}{v_{n}^{4} t_{0}^{2} \cos { }^{6} \theta}+\ldots
\end{aligned}
$$

Note that the series (5) for a TTI medium contains both even and odd power terms. Each Taylor series coefficient in (5) is given by the series with the number of terms that corresponds to the number of terms in the truncated Taylor series (4).

Applying the transformation on the hyperbolic approximation

$$
t^{2}(x)=t_{0}^{2}+\frac{x^{2}}{v_{n}^{2}}
$$

yields the hyperbola with shifted apex position,

$$
t_{n}^{2}\left(x_{n}\right)=t_{0 n}^{2}+\frac{\left(x_{n}-x_{0 n}\right)^{2}}{v_{n, n}^{2}} \text {, }
$$

where the new traveltime parameters are given by

$$
\begin{aligned}
& t_{0 n}^{2}=t_{0}^{2} \frac{v_{0}^{2}}{v_{0}^{2} \cos ^{2} \theta+v_{n}^{2} \sin ^{2} \theta} \\
& x_{0 n}=\frac{t_{0} v_{0}\left(v_{0}^{2}-v_{n}^{2}\right) \sin \theta \cos \theta}{v_{0}^{2} \cos ^{2} \theta+v_{n}^{2} \sin ^{2} \theta} . \\
& v_{n, n}^{2}=\frac{v_{0}^{2} v_{n}^{2}}{v_{0}^{2} \cos ^{2} \theta+v_{n}^{2} \sin ^{2} \theta}
\end{aligned}
$$

The moveout approximation (7) is valid for elliptical TTI media (Golikov and Stovas, 2012).

Applying the transformation on the rational approximation shown in Alkhalifah and Tsvankin (1995) as,

$$
t^{2}(x)=t_{0}^{2}+\frac{x^{2}}{v_{n}^{2}}-\frac{2 \eta x^{4}}{v_{n}^{4} t_{0}^{2}\left(1+(1+2 \eta) \frac{x^{2}}{v_{n}^{2} t_{0}^{2}}\right)},
$$

$$
t_{n}^{2}\left(x_{n}\right)=\frac{a_{0}+a_{1} x_{n}+a_{2} x_{n}^{2}+a_{3} x_{n}^{3}+a_{4} x_{n}^{4}}{b_{0}+b_{1} x_{n}+b_{2} x_{n}^{2}}
$$

with parameters

$$
\begin{aligned}
& a_{0}=v_{0}^{4} t_{0}^{4}\left(v_{n}^{4} \cos ^{4} \theta+2 v_{0}^{2} v_{n}^{2}(1+\eta) \sin ^{2} \theta \cos ^{2} \theta+v_{0}^{4} \sin ^{4} \theta\right) \\
& a_{1}=4 v_{0}^{3} t_{0}^{3} \cos \theta \sin \theta\left(v_{n}^{4} \cos ^{2} \theta-v_{0}^{2} v_{n}^{2}(1+\eta) \cos 2 \theta-v_{0}^{4} \sin ^{2} \theta\right) \\
& a_{2}=2 v_{0}^{2} t_{0}^{2}\left(3 v_{n}^{4} \sin ^{2} \theta \cos ^{2} \theta+v_{0}^{2} v_{n}^{2}(1+\eta)\left(1-6 \sin ^{2} \theta \cos ^{2} \theta\right)\right. \\
& \left.+3 v_{0}^{4} \sin ^{2} \theta \cos ^{2} \theta\right) \\
& a_{3}=4 v_{0} t_{0} \cos \theta \sin \theta\left(v_{n}^{4} \sin ^{2} \theta+v_{0}^{2} v_{n}^{2}(1+\eta) \cos 2 \theta-v_{0}^{4} \cos ^{2} \theta\right) \\
& a_{4}=v_{n}^{4} \sin ^{4} \theta+2 v_{0}^{2} v_{n}^{2}(1+\eta) \sin ^{2} \theta \cos ^{2} \theta+v_{0}^{4} \cos ^{4} \theta \\
& b_{0}=v_{0}^{4} v_{n}^{2} t_{0}^{2}\left(v_{n}^{2} \cos ^{2} \theta+(1+2 \eta) v_{0}^{2} \sin ^{2} \theta\right) \\
& b_{1}=v_{0}^{3} v_{n}^{2} t_{0} \sin 2 \theta\left(v_{n}^{2}-(1+2 \eta) v_{0}^{2}\right) \\
& b_{2}=v_{0}^{2} v_{n}^{2}\left(v_{n}^{2} \sin ^{2} \theta+(1+2 \eta) v_{0}^{2} \cos ^{2} \theta\right)
\end{aligned}
$$

For the shifted hyperbola equation (Malovichko, 1978)

$$
t(x)=t_{0}\left[1+\frac{1}{S_{2}}\left(\sqrt{1+S_{2} \frac{x^{2}}{v_{n}^{2} t_{0}^{2}}}-1\right)\right] \text {, }
$$

we obtain

$$
\begin{aligned}
& t_{n}\left(x_{n}\right)=\frac{1}{S_{2} v_{0} v_{n}}\left[\left(S_{2}-1\right) v_{n}\left(x_{n} \cos \theta+v_{0} t_{0} \sin \theta\right)\right. \\
& \left.+\sqrt{S_{2} v_{0}^{2}\left(x_{n} \cos \theta-v_{0} t_{0} \sin \theta\right)^{2}+v_{n m o}^{2}\left(x_{n} \sin \theta+v_{0} t_{0} \cos \theta\right)^{2}}\right]
\end{aligned}
$$

We can map any of analytical moveout approximation from VTI to TTI media including the most accurate one, the generalized approximation.

\section{Analysis of the approximations}

Now we can analyze the vertical $\left(x_{n}=0\right)$ and horizontal $\left(x_{n} \rightarrow \infty\right)$ velocities from the mapped moveout approximations.

From the hyperbolic approximation, we obtain

$$
\begin{aligned}
& \frac{1}{V_{0}^{2}}=\frac{\cos ^{2} \theta}{v_{0}^{2}}+\frac{\sin ^{2} \theta}{v_{n}^{2}} \\
& \frac{1}{V_{x}^{2}}=\frac{\cos ^{2} \theta}{v_{n}^{2}}+\frac{\sin ^{2} \theta}{v_{0}^{2}}
\end{aligned}
$$

yields another rational approximation 


\section{Mapping of moveout approximations in TI media}

$$
\begin{gathered}
\frac{1}{V_{0}^{2}}=\frac{\frac{\cos ^{4} \theta}{v_{0}^{4}}+2(1+\eta) \frac{\sin ^{2} \theta \cos ^{2} \theta}{v_{0}^{2} v_{n}^{2}}+\frac{\sin ^{4} \theta}{v_{n}^{4}}}{\frac{\cos ^{2} \theta}{v_{0}^{2}}+(1+2 \eta) \frac{\sin ^{2} \theta}{v_{n}^{2}}} \\
\frac{1}{V_{x}^{2}}=\frac{\frac{\sin ^{4} \theta}{v_{0}^{4}}+2(1+\eta) \frac{\sin ^{2} \theta \cos ^{2} \theta}{v_{0}^{2} v_{n}^{2}}+\frac{\cos ^{4} \theta}{v_{n}^{4}}}{\frac{\sin ^{2} \theta}{v_{0}^{2}}+(1+2 \eta) \frac{\cos ^{2} \theta}{v_{n}^{2}}}
\end{gathered}
$$

For shifted hyperbola approximation, we have

$$
\begin{aligned}
& \frac{1}{V_{0}}=\frac{1}{S_{2}}\left[\frac{\left(S_{2}-1\right) \sin \theta}{v_{0}}+\sqrt{\frac{\cos ^{2} \theta}{v_{0}^{2}}+\frac{S_{2} \sin ^{2} \theta}{v_{n}^{2}}}\right] \\
& \frac{1}{V_{x}}=\frac{1}{S_{2}}\left[\frac{\left(S_{2}-1\right) \cos \theta}{v_{0}}+\sqrt{\frac{\sin ^{2} \theta}{v_{0}^{2}}+\frac{S_{2} \cos ^{2} \theta}{v_{n}^{2}}}\right]
\end{aligned}
$$

For the generalized moveout approximation, we obtain

$$
\begin{aligned}
& \frac{1}{V_{0}^{2}}=\frac{\cos ^{2} \theta}{v_{0}^{2}}+\frac{\sin ^{2} \theta}{v_{n}^{2}} \\
& -\frac{4 \eta \frac{\sin ^{4} \theta}{v_{n}^{4}}}{\frac{\cos ^{2} \theta}{v_{0}^{2}}+B \frac{\sin ^{2} \theta}{v_{n}^{2}}+\sqrt{\frac{\cos ^{4} \theta}{v_{0}^{4}}+2 B \frac{\sin ^{2} \theta \cos ^{2} \theta}{v_{0}^{2} v_{n}^{2}}+C \frac{\sin ^{4} \theta}{v_{n}^{4}}}} \\
& \frac{1}{V_{x}^{2}}=\frac{\cos ^{2} \theta}{v_{n}^{2}}+\frac{\sin ^{2} \theta}{v_{0}^{2}} \\
& -\frac{\cos ^{2} \theta}{v_{n}^{2}}+B \frac{\sin ^{2} \theta}{v_{0}^{2}}+\sqrt{\frac{\cos ^{4} \theta}{v_{n}^{4}}+2 B \frac{\cos ^{4} \theta}{v_{n}^{4}}}
\end{aligned}
$$

The parameters $B$ and $C$ are calculated from a horizontal ray in a homogeneous VTI medium and equal to $\left(1+8 \eta+8 \eta^{2}\right) /(1+2 \eta)$ and $1 /(1+2 \eta)^{2}$, respectively (Fomel and Stovas, 2010).

Due to the symmetry of the TTI model over $\pi / 2$ all equations for the horizontal velocity can be obtained from the equations for the vertical velocity by interchanging of sine and cosine functions. The normal moveout velocities, which are crucial for surface seismic data, defined at $x_{n}=0$, can also be easily computed from the mapped moveout approximations.
The approximated vertical, horizontal and normal moveout velocities are plotted in Figure 1 versus the tilt angle for anisotropic medium with parameters: $v_{0}=2.0 \mathrm{~km} / \mathrm{s}$, $\delta=0.1$ and $\eta=0.2$. We can see that the results from the hyperbolic and the shifted hyperbola approximations, especially for the normal moveout velocity, are very different from the ones obtained from the rational and generalized approximations.

\section{Numerical examples}

To illustrate the accuracy of the proposed approximation, we select a homogeneous TTI model with parameters

mentioned above and $\theta=30^{\circ}$. In Figure 2, we display the relative error in traveltime obtained from selected moveout approximations being mapped from a VTI medium. The generalized approximation gives the best results with a maximum relative error of about 0.0002 on the offset/depth spread up to 5 . The worst results are obtained from a hyperbolic approximation. Note that at offset $x_{n}=z \tan \theta$, which corresponds to $x=0$, all moveout approximations predict the exact traveltime. In Figure 3, we show the relative error in traveltime for mapped generalized approximation for different values of symmetry tilt angles. We can see that the accuracy of the generalized moveout approximation remains the same regardless to the symmetry direction.

\section{Layered media}

For layered media with a constant tilt in the symmetry axis (factorized anisotropic in the tilt), effective media approximations used for the VTI equations holds here as well. Nevertheless, the parameters now are those corresponding to the symmetry axis direction. For example the vertical velocity would be given by

$v_{\text {oeff }}\left(t_{0}\right)=\frac{1}{t_{0}} \int_{0}^{t_{0}} v_{0}(\tau) d \tau$,

where $v_{0}\left(t_{0}\right)$ is the velocity along the constant symmetry direction. The same applies for other parameters.

\section{Conclusions}

We develop an analytical general formula to map moveout approximations from VTI to TTI media. The method is applied on the hyperbolic, the shifted hyperbola, the rational and the generalized approximations for a vertical symmetry axis direction. The mapped moveout approximations are also used to derive approximations for vertical and horizontal velocities. 


\section{Mapping of moveout approximations in TI media}

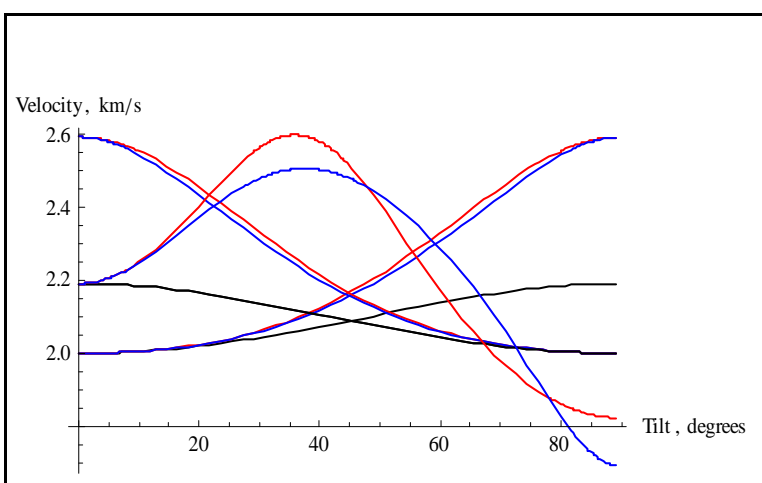

Figure 1: The vertical, horizontal and normal moveout velocities computed from the mapped moveout approximations for a homogeneous TTI model is given by $v_{0}=2.0 \mathrm{~km} / \mathrm{s}, \delta=0.1$ and $\eta=0.2$. The hyperbolic, rational and generalized moveout approximations are indicated by black, red and blue lines, respectively. Note, that for hyperbolic moveout approximation, the horizontal and normal moveout velocities coincide. No results are shown for the shifted hyperbola approximation since it is not suitable for a homogeneous VTI or TTI model.

The accuracy of the mapped moveout approximations is tested for a homogeneous TTI medium example. We show that the generalized moveout approximation being mapped to a TTI medium remains the most accurate one.

\section{Acknowledgments}

Alexey Stovas would like to acknowledge the ROSE project for financial support. Tariq Alkhalifah would like to thank KAUST for its financial support. 
http://dx.doi.org/10.1190/segam2012-0050.1

\section{EDITED REFERENCES}

Note: This reference list is a copy-edited version of the reference list submitted by the author. Reference lists for the 2012 SEG Technical Program Expanded Abstracts have been copy edited so that references provided with the online metadata for each paper will achieve a high degree of linking to cited sources that appear on the Web.

\section{REFERENCES}

Alkhalifah, T., 2011a, Scanning anisotropy parameters in complex media: Geophysics, 76, no. 2, U13$\mathrm{U} 22$.

Alkhalifah, T., 2011b, Traveltime approximations for transversely isotropic media with an inhomogeneous background: Geophysics, 76, no. 3, WA31-WA42.

Alkhalifah, T., and I. Tsvankin, 1995, Velocity analysis for transversely isotropic media: Geophysics, 60 , $1550-1566$.

Fomel, S., and A. Stovas, 2010, The generalized non-hyperbolic moveout approximation: Geophysics, 75, no. 2, N2, U9-U18.

Golikov, P., and A. Stovas, 2012, Traveltime parameters in a tilted elliptical anisotropic medium: Geophysical Prospecting, in press.

Malovichko, A. A., 1978, A new representation of the traveltime curve of reflected waves in horizontally layered media: Applied Geophysics in Russian, 91, 47-53. English translation in C. H. Sword, 1987, A Soviet look at datum shift, SEP-51: SEP, 313-316, accessed 26 January 2010; http://sepwww.stanford.edu/data/media/public/oldreports/sep51/51_22.pdf.

Pech, A., I. Tsvankin, and V. Grechka, 2003, Quartic moveout coefficient: 3D description and application to tilted TI media: Geophysics, 68, 1600-1610.

Stovas, A., and T.Alkhalifah, 2011, A new traveltime approximation for TI media: Presented at the $73^{\text {rd }}$ Annual International Conference and Exhibition, EAGE.

Stovas, A., and T. Alkhalifah, 2012, Mapping of moveout in TTI media:Presented at the $74^{\text {th }}$ Annual International Conference and Exhibition, EAGE,. 